\title{
Effect of Osteopathic Manipulative Treatment vs Sham Treatment on Activity Limitations in Patients With Nonspecific Subacute and Chronic Low Back Pain A Randomized Clinical Trial
}

Christelle Nguyen, MD, PhD; Isabelle Boutron, MD, PhD; Rafael Zegarra-Parodi; Gabriel Baron, PhD; Sophie Alami, PhD; Katherine Sanchez, MD; Camille Daste, MD, MSc; Margaux Boisson, MD; Laurent Fabre; Peggy Krief, MD; Guillaume Krief; Marie-Martine Lefèvre-Colau, MD, PhD; François Rannou, MD, PhD

IMPORTANCE Osteopathic manipulative treatment (OMT) is frequently offered to people with nonspecific low back pain (LBP) but never compared with sham OMT for reducing LBP-specific activity limitations.

OBJECTIVE To compare the efficacy of standard OMT vs sham OMT for reducing LBP-specific activity limitations at 3 months in persons with nonspecific subacute or chronic LBP.

DESIGN, SETTING, AND PARTICIPANTS This prospective, parallel-group, single-blind, single-center, sham-controlled randomized clinical trial recruited participants with nonspecific subacute or chronic LBP from a tertiary care center in France starting February 17, 2014, with follow-up completed on October 23, 2017. Participants were randomly allocated to interventions in a 1:1 ratio. Data were analyzed from March 22, 2018, to December 5, 2018.

INTERVENTIONS Six sessions (1 every 2 weeks) of standard OMT or sham OMT delivered by nonphysician, nonphysiotherapist osteopathic practitioners.

MAIN OUTCOMES AND MEASURES The primary end point was mean reduction in LBP-specific activity limitations at 3 months as measured by the self-administered Quebec Back Pain Disability Index (score range, 0-100). Secondary outcomes were mean reduction in LBP-specific activity limitations; mean changes in pain and health-related quality of life; number and duration of sick leaves, as well as number of LBP episodes at 12 months; and consumption of analgesics and nonsteroidal anti-inflammatory drugs at 3 and 12 months. Adverse events were self-reported at 3, 6, and 12 months.

RESULTS Overall, 200 participants were randomly allocated to standard OMT and 200 to sham OMT, with 197 analyzed in each group; the median (range) age at inclusion was 49.8 (40.7-55.8) years, 235 of 394 (59.6\%) participants were women, and 359 of 393 (91.3\%) were currently working. The mean (SD) duration of the current LBP episode was 7.5 (14.2) months. Overall, 164 (83.2\%) patients in the standard OMT group and 159 (80.7\%) patients in the sham OMT group had the primary outcome data available at 3 months. The mean (SD) Quebec Back Pain Disability Index scores for the standard OMT group were 31.5 (14.1) at baseline and 25.3 (15.3) at 3 months, and in the sham OMT group were 27.2 (14.8) at baseline and 26.1 (15.1) at 3 months. The mean reduction in LBP-specific activity limitations at 3 months was $-4.7(95 \% \mathrm{Cl},-6.6$ to -2.8$)$ and $-1.3(95 \% \mathrm{Cl},-3.3$ to 0.6$)$ for the standard OMT and sham OMT groups, respectively (mean difference, $-3.4 ; 95 \% \mathrm{Cl},-6.0$ to $-0.7 ; P=.01$ ). At 12 months, the mean difference in mean reduction in LBP-specific activity limitations was $-4.3(95 \% \mathrm{Cl},-7.6$ to $-1.0 ; P=.01)$, and at 3 and 12 months, the mean difference in mean reduction in pain was $-1.0(95 \% \mathrm{Cl},-5.5$ to $3.5 ; P=.66)$ and $-2.0(95 \% \mathrm{Cl},-7.2$ to $3.3 ; P=.47)$, respectively. There were no statistically significant differences in other secondary outcomes. Four and 8 serious adverse events were self-reported in the standard OMT and sham OMT groups, respectively, though none was considered related to OMT.

CONCLUSIONS AND RELEVANCE In this randomized clinical trial of patients with nonspecific subacute or chronic LBP, standard OMT had a small effect on LBP-specific activity limitations vs sham OMT. However, the clinical relevance of this effect is questionable.

TRIAL REGISTRATION ClinicalTrials.gov Identifier: NCT02034864

JAMA Intern Med. doi:10.1001/jamainternmed.2021.0005

Published online March 15, 2021.
Visual Abstract

Supplemental content
Author Affiliations: Author affiliations are listed at the end of this article.

Corresponding Author: Christelle Nguyen, MD, PhD, AP-HP, Centre-Université de Paris, Hôpital Cochin, Service de Rééducation et de Réadaptation de l'Appareil Locomoteur et des Pathologies du Rachis, 27, Rue du Faubourg Saint-Jacques, 75014 Paris, France (christelle.nguyen2@aphp.fr). 
$\mathrm{N}$ onspecific low back pain (LBP) is the major cause of years lived with disability in the world. ${ }^{1}$ Low back pain is usually treated according to symptom duration, presence of concomitant radicular pain, and consistent anatomical abnormalities, ${ }^{2}$ as well as patient phenotype. ${ }^{3}$ The prognosis of acute LBP is good, ${ }^{4}$ but in $5 \%$ to $15 \%$ of cases, LBP becomes chronic. ${ }^{5}$ Current therapeutic strategies are stratified according to risk of persistent disability in the long term. ${ }^{6}$ They include pharmacological and nonpharmacological treatments, such as manual therapy, exercise therapy, education, selfmanagement, cognitive behavioral therapy, or multidisciplinary rehabilitation. ${ }^{7}$

Osteopathic manipulative treatment (OMT) is a manual therapy that belongs to the category of complementary and alternative medicines practiced outside of the United States. In France, osteopathic practice is shared between different professionals, including nonmedical and non-health professionals. Nonphysician, nonphysiotherapist osteopathic practitioners represent about $60 \%$ of osteopathic practitioners, according to French usage. ${ }^{8}$ Contrary to biomedical approaches in manual therapies applying lumbar spinal manipulative treatments for LBP, ${ }^{9,10}$ osteopathic treatment for LBP offers spinal manipulative treatments and nonspinal manipulative treatments applied to both symptomatic and distant nonsymptomatic regions. ${ }^{11}$ Recent bodies of evidence suggest effects of manual therapies related to improvement of body perception may reduce anxiety, avoidance, and defensive musculoskeletal responses. ${ }^{12}$

In acute LBP, manipulative treatment modestly reduces pain and activity limitations up to 6 weeks. ${ }^{13}$ Whether these effects are explained by manipulative treatment as a standalone intervention or as part of a bundle of therapies is unclear. ${ }^{13}$ In subacute and chronic LBP, ${ }^{10,14,15}$ findings are inconsistent, and comparisons of standard manipulative treatment with sham manipulative treatment are lacking. ${ }^{9,10}$ Focusing specifically on OMT, Franke and colleagues ${ }^{11}$ and the American Osteopathic Association ${ }^{16}$ call for larger randomized clinical trials (RCTs). In the present study, we aimed to compare the efficacy of standard OMT with sham OMT for reducing LBP-specific activity limitations at 3 months in nonspecific subacute or chronic LBP.

\section{Methods}

\section{Design Overview}

We conducted a prospective, parallel-group, randomized, single-blind, single-center, sham-controlled trial. Recruitment started on February 17, 2014, and follow-up was completed on October 23, 2017. Patient written and verbal informed consent was obtained from all participants by the investigator during a face-to-face inclusion visit. The study was approved by the Comité de Protection des Personnes île-deFrance III and followed Consolidated Standards of Reporting Trials (CONSORT) reporting guidelines. The full original and final protocols are provided in Supplement 1. Substantial changes made to the methods after the trial commencement are detailed in eAppendix 1 in Supplement 2 . We did not switch

\section{Key Points}

Question What is the effectiveness of osteopathic manipulative treatment (OMT) compared with sham OMT in reducing low back pain (LBP)-specific activity limitations in people with nonspecific subacute and chronic LBP?

Findings In this randomized clinical trial that included 400 participants, standard OMT had a small effect on LBP-specific activity limitations vs sham OMT at 3 months. However, this effect was likely not clinically meaningful.

Meaning These results raise the issue of the usefulness of OMT in people with nonspecific subacute and chronic LBP.

outcomes after trial completion. Amendments made to the trial registry after trial completion were only aimed at providing a more accurate definition of outcomes. All were completed blind to the data.

\section{Blinding}

Investigators and osteopathic practitioners were not blinded, but data analysts and participants were blinded. Blinding is challenging to achieve in nonpharmacological trials but is important because unblinding of participants could have an effect on patient-reported outcomes of musculoskeletal conditions. ${ }^{17}$ To maintain the blinding of participants, we implemented 3 measures ${ }^{18}$ : (1) we designed a sham control that consisted of light touch, an a priori inert procedure used to reduce therapeutic aspect of the touch by the osteopathic practitioner while maintaining a similar manual contact time in both groups; despite some limitations, ${ }^{19}$ light touch has been proposed to simulate OMT without simulating physiotherapy or massage $^{2 \mathrm{O}}$; (2) osteopathic practitioners were not allowed to have contact with participants outside of the sessions; and (3) study announcements and information delivered remained neutral and refrained from using the word osteopathy, rather referring to manual therapy for both interventions.

To ascertain that the osteopathic practitioners' speech was consistent in both groups, OMT sessions were audio recorded. Two sociologists (including S.A.) qualitatively assessed 60 randomly selected audio records (30 from each group). They used 23 items to assess the duration of the sessions, the respect of the recommendations for verbal behavior, the content of the speech, and the verbal attitude. Scores calculated from 20 out of 23 items are summarized in 6 domains: instrumental exchanges, listening quality, personal exchanges, quality of dialogue, reassurance, and relational proximity (eAppendix 2 in Supplement 2). To ensure no imbalance in the credibility of the interventions and expectancies of the participants, the Credibility/Expectancy Questionnaire ${ }^{21,22}$ was self-administered at 3 months.

\section{Setting and Participants}

The study was conducted in the physical medicine and rehabilitation department of a tertiary care center in France. Participants were recruited among inpatients and outpatients of the department. A campaign targeting employees with chronic LBP in the Assistance Publique-Hôpitaux de Paris, a French 
public health institution that employs approximately 100000 people in 39 hospitals, was also conducted. People who were interested in participating in the study were invited to call a management center, which screened for eligibility, then scheduled the baseline face-to-face visit with the investigator, a board-certified specialist in physical medicine and rehabilitation and/or rheumatology. Inclusion criteria were patients who were male or female sex, had nonspecific subacute or chronic LBP (ie, duration of LBP $\geq 6$ weeks), were aged 18 to 65 years, who could speak and understand French, who gave informed consent to participate in the study, and who were affiliated with or beneficiary of social insurance. Exclusion criteria were specific LBP caused by inflammatory, tumoral, infectious disease, or a back traumatism in the past 3 months; history of back surgery and/or vertebral fracture in the previous 6 months; presence of a motor impairment related to the reason for consultation; pregnancy; inability to understand the process of the study; and patients who were students or practitioners in manipulative treatments or who were already included in another clinical study. Patients who had concomitant radicular syndromes (eg, sciatica) or who had previous experience with manipulative therapy were not excluded.

\section{Randomization and Interventions}

Randomization was centralized, and participants were randomly allocated in a 1:1 ratio. An independent statistician provided a computer-generated randomization list with permuted blocks of variable sizes (2, 4 , and 6$)$. The sequence was concealed by use of a computer interface implemented in the electronic case report form. At baseline, the investigator collected baseline characteristics and randomly allocated participants to 1 of the 2 groups. A clinical research technician scheduled appointments for OMT sessions.

The first OMT session was scheduled approximately within 2 weeks after randomization. The experimental group received 6 sessions of standard OMT, and the control group 6 sessions of sham OMT, each session at 2 -week intervals. For both experimental and control groups, each session lasted 45 minutes and consisted of 3 periods: (1) interview focusing on pain location, (2) full osteopathic examination, and (3) intervention consisting of standard or sham OMT. Briefly, in both groups, practitioners assessed 7 anatomical regions for dysfunction (lumbar spine, root of mesentery, diaphragm, and atlantooccipital, sacroiliac, temporomandibular, and talocrural joints) and applied sham OMT to all areas or standard OMT to those that were considered dysfunctional. All health care providers were board-certified nonphysician, nonphysiotherapist osteopathic practitioners (Répertoire National de la Certification Professionnelle, niveau 1). They all received a 2-day training according to international standards to deliver both standard and sham OMT. ${ }^{23}$ Full descriptions of osteopathic practitioner training and interventions are provided in eAppendices 3 and 4 in Supplement 2. ${ }^{24,25}$ In both groups, pharmacological interventions, nonpharmacological interventions, and spinal surgery were allowed. Cointerventions were self-reported at 3, 6, and 12 months by use of a standardized checklist (eAppendix 5 in Supplement 2).
Outcomes and Follow-up

Self-administered questionnaires assessing primary and secondary outcomes were collected during face-to-face follow-up visits. If the participant could not attend the visits, questionnaires were collected by mail or over telephone by a clinical research technician. All primary and secondary efficacy outcomes were prespecified in the full protocol and are reported in the present article.

The primary efficacy outcome was the mean reduction from baseline in LBP-specific activity limitations at 3 months after randomization, assessed via the self-administered Quebec Back Pain Disability Index (QBPDI), with scores ranging from 0 (no limitations) to 100 (maximum limitations). ${ }^{26-28}$ Secondary efficacy outcomes were mean reduction from baseline in LBP-specific activity limitations at 12 months, assessed via the self-administered QBPDI; mean reduction from baseline in pain in the previous 48 hours, assessed via a selfadministered 11-class numeric rating scale, with scores ranging from 0 (no pain) to 100 (maximum pain) in 10-point increments at 3 and 12 months; mean improvement from baseline in health-related quality of life (HRQOL), assessed via the selfadministered Medical Outcomes Study Short Form 12, the physical component summary ranging in score from 9.95 (worse HRQOL) to 70.02 (best HRQOL), and mental component summary ranging in score from 5.89 (worse HRQOL) to 71.97 (best HRQOL), both at 3 and 12 months; self-reported number and duration (days) of sick leaves at 12 months; selfreported number of LBP episodes at 12 months; and selfreported consumption of analgesics and nonsteroidal antiinflammatory drugs since last contact at 3 and 12 months.

Safety outcomes were recorded at 3, 6, and 12 months by using an open-ended question ("Did you have any adverse events?"). Serious adverse events were reviewed by 2 independent blinded investigators (C.D. and M.B.). Events were classified into 6 categories: (1) hospitalization for usual care of LBP, (2) hospitalization for another condition, (3) hospitalization related to the intervention received, (4) usual care (without hospitalization) of LBP, (5) usual care (without hospitalization) of another condition, and (6) usual care (without hospitalization) related to the intervention received.

\section{Statistical Analysis}

The original and final versions of the statistical analysis plan are available in Supplement 3. With an a risk of .05, a power $(1-\beta)$ of .90 , and a predicted mean difference in mean (SD) change of QBPDI score at 3 months of 7 (20) points, which corresponds to an effect size of 0.35 , we needed 173 patients in each group. Estimating that $10 \%$ of patients would be lost to follow-up, we sought to include 200 patients in each group. Categorical variables are described with frequencies and percentages. Quantitative variables are described with mean (SD) or median (first and third quartiles). To compare between-group differences in mean change from baseline for quantitative outcomes, we used a constrained longitudinal data analysis. ${ }^{29-31}$ This mixed model is a constrained fulllikelihood approach, whereby both the baseline and postbaseline values are modeled as dependent variables (the constrained longitudinal data analysis model assumes that both 


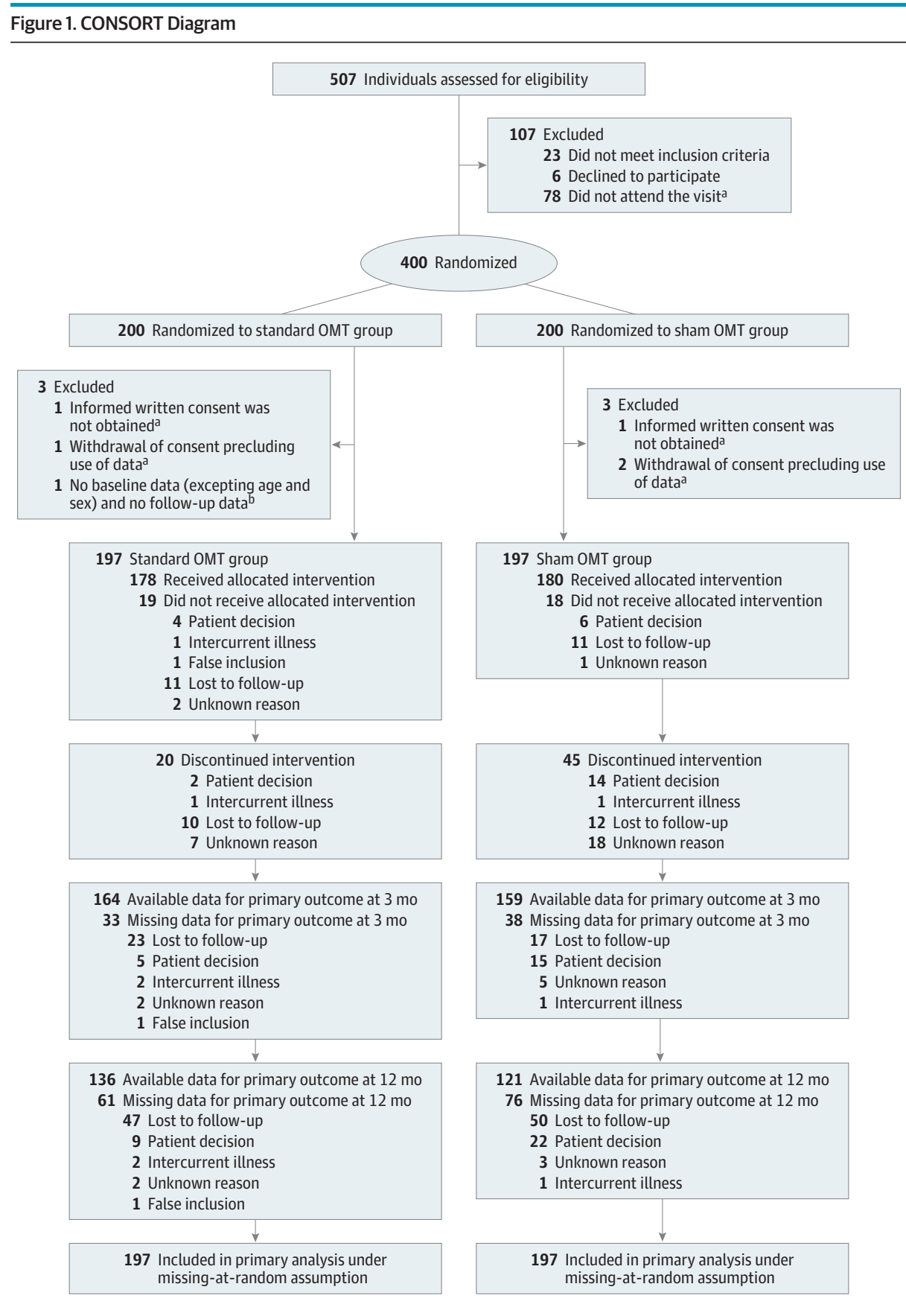

OMT indicates osteopathic manipulative treatment.

according to French regulation, no data were analyzed for these patients.

${ }^{\mathrm{b}}$ This patient did not receive allocated intervention. the baseline and postbaseline measurements are jointly multivariate normally distributed because the baseline value is treated as part of the response vector). The true baseline means are constrained to be the same for the 2 treatment groups. The constrained longitudinal data analysis model can include all randomized participants with at least 1 baseline or postbaseline value. Such methods based on maximum likelihood are consistent under the missing-at-random assumption (ie, the probability of dropout is related to some observable characteristics or quantity such as treatment arm assignment). Hence, this analysis provides an adjustment for the observed baseline difference in estimating the treatment. A random effect at the participant level was included in these models. Results are expressed as differences in mean change from baseline with 95\% CIs at 3 and 12 months postrandomization.

As a post hoc sensitivity analysis requested by peer reviewers to improve results interpretation, we report the responder rate for the minimal important change, defined as an absolute change of at least 20 points in QBPDI score according to Ostelo and colleagues. ${ }^{32}$ We also report a cumulative proportion of responders analysis graph in patients with available QBPDI data at 3 months. ${ }^{33}$ Percentage of responders over a range of possible cutoffs points of the QBPDI score change are displayed in this graph. Number needed to treat was also 
Table 1. Baseline Characteristics and Sessions of Patients With Nonspecific Subacute and Chronic Low Back Pain (LBP) Receiving Standard and Sham Osteopathic Manipulative Treatment (OMT) ${ }^{\mathrm{a}}$

\begin{tabular}{|c|c|c|}
\hline \multirow[b]{2}{*}{ Characteristic } & \multicolumn{2}{|c|}{ No./total reported (\%) } \\
\hline & $\begin{array}{l}\text { Standard group } \\
(\mathrm{n}=197)\end{array}$ & Sham group $(n=197)$ \\
\hline Age, mean (SD), y & $48.3(11.9)$ & $47.5(10.6)$ \\
\hline Female & $116 / 197(58.9)$ & $119 / 197(60.4)$ \\
\hline Completed higher education & $132 / 196(67.3)$ & $128 / 197(65.0)$ \\
\hline Currently working & $175 / 196(89.3)$ & $184 / 197(93.4)$ \\
\hline Chronic LBP duration, mean (SD), y & $14.2(10.8)$ & $14.2(10.6)$ \\
\hline Total reported & 195 & 196 \\
\hline Current LBP episode duration, mean (SD), mo & $6.5(10.3)$ & $8.5(17.2)$ \\
\hline Total reported & 194 & 196 \\
\hline LBP pain intensity on NRS (range, 1-100), mean (SD) ${ }^{\mathrm{b}}$ & $42.0(21.1)$ & $41.4(21.5)$ \\
\hline Total reported & 193 & 194 \\
\hline Radicular pain intensity on NRS (range, $1-100$ ), mean (SD) ${ }^{b}$ & $19.2(21.7)$ & $15.5(21.6)$ \\
\hline Total reported & 193 & 194 \\
\hline QBPDI score (range, 1-100), mean (SD) ${ }^{c}$ & $31.5(14.1)$ & $27.2(14.8)$ \\
\hline Total reported & 197 & 196 \\
\hline \multicolumn{3}{|l|}{ MOS SF-12 score, mean (SD) ${ }^{d}$} \\
\hline Physical component summary (range, 9.95-70.02) & $40.2(8.2)$ & $41.9(7.8)$ \\
\hline Total reported & 195 & 194 \\
\hline Mental component summary (range, $5.89-71.97$ ) & $42.9(10.9)$ & $42.7(10.7)$ \\
\hline Total reported & 195 & 194 \\
\hline \multicolumn{3}{|l|}{ Main anatomical finding } \\
\hline Lumbar spinal stenosis & $4 / 196(2.0)$ & $3 / 196(1.5)$ \\
\hline Active discopathy & $10 / 196(5.1)$ & $8 / 196(4.1)$ \\
\hline Disk herniation & $10 / 196(5.1)$ & $8 / 196(4.1)$ \\
\hline Scheuermann disease & $2 / 196(1.0)$ & $5 / 196(2.6)$ \\
\hline Degenerative disk disease & $86 / 196(43.9)$ & $62 / 196(31.6)$ \\
\hline Facet joint osteoarthritis & $16 / 196(8.2)$ & $13 / 196(6.6)$ \\
\hline Spondylolisthesis & $5 / 196(2.6)$ & $1 / 196(0.5)$ \\
\hline Lumbar scoliosis & $9 / 196(4.6)$ & $21 / 196(10.7)$ \\
\hline Undetermined & $53 / 196(27.0)$ & $73 / 196(37.2)$ \\
\hline Other & $1 / 196(0.5)$ & 2/196 (1.0) \\
\hline \multicolumn{3}{|l|}{ Previous treatments } \\
\hline Analgesics & $173 / 188(92.0)$ & $172 / 193(89.1)$ \\
\hline Nonopioids & $167 / 188(88.8)$ & $170 / 193(88.1)$ \\
\hline Weak opioids ${ }^{\mathrm{e}}$ & $124 / 188(66.0)$ & $113 / 193(58.5)$ \\
\hline Strong opioids ${ }^{\mathrm{e}}$ & $21 / 188(11.2)$ & $17 / 193(8.8)$ \\
\hline Nonsteroidal anti-inflammatory drugs & $160 / 188(85.1)$ & $157 / 193(81.3)$ \\
\hline Muscle relaxants & $119 / 188(63.3)$ & 106/193 (54.9) \\
\hline Anxiolytics & 25/188 (13.3) & 20/193 (10.4) \\
\hline Antidepressants & $19 / 188(10.1)$ & 21/193 (10.9) \\
\hline Antiepileptics & $15 / 188(8.0)$ & $8 / 193(4.1)$ \\
\hline Spinal corticosteroid injections & $55 / 188(29.3)$ & $38 / 193(19.7)$ \\
\hline Lumbar brace & $125188(66.5)$ & $119 / 193(61.7)$ \\
\hline Outpatient physiotherapy & $146 / 188(77.7)$ & $140 / 193(72.5)$ \\
\hline Multidisciplinary rehabilitation & $15 / 188(8.0)$ & 22/193 (11.4) \\
\hline Alternative medicine & $100 / 188(53.2)$ & $104 / 193(53.9)$ \\
\hline
\end{tabular}

(continued) 


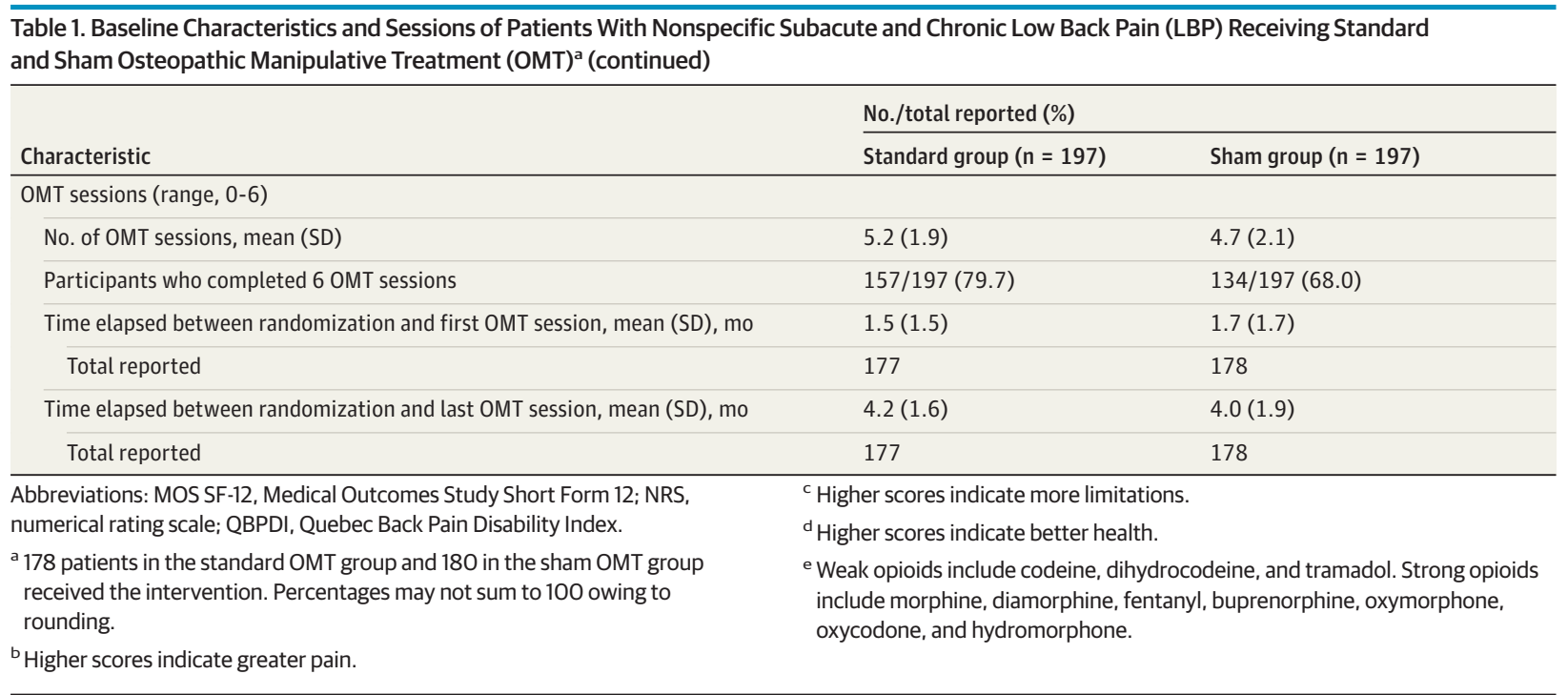

computed for a change of at least 20 points in QBPDI score. ${ }^{34,35}$ A Poisson model was used to estimate between-group differences for consumption of analgesics and nonsteroidal antiinflammatory drugs at 3 and 12 months postrandomization. Results are expressed as absolute risk difference, relative risk, and 95\% CI (the delta method was used for computing the 95\% CI for absolute risk difference). Negative binomial regression with an offset term (duration of follow-up) was used to estimate between-group differences for sick leave duration, number of sick leaves, and number of LBP episodes at 3 and 12 months postrandomization. Results are expressed as absolute mean difference and ratio of means and 95\% CIs. Safety outcomes were described in each group. All statistical tests were 2-sided, and $P<.05$ was considered statistically significant. Data were analyzed by using SAS, version 9.3 (SAS Institute Inc), with the procedures MIXED (constrained longitudinal data analysis model) and GENMOD (negative binomial regression). The Poisson model was performed with the glm function of $\mathrm{R}$, version 4.1.1 (R Foundation for Statistical Computing).

\section{Results}

Overall, 507 people were screened, and 400 participants were randomly assigned to receive standard OMT $(n=200)$ or sham OMT ( $\mathrm{n}=200$ ). After randomization, 5 randomized patients (2 in the standard OMT group and 3 in the sham OMT group) were excluded from all analysis; no informed consent was obtained for 2 patients, and 3 patients withdrew their consent with no possibility to use their data. According to the French Public Health Code applicable to research involving humans, data of these 5 patients were erased. A sixth randomized patient (in the standard OMT group) was excluded owing to no baseline data (excepting age and sex) and no follow-up data. Overall, 197 participants in the standard OMT group and 197 in the sham OMT group were considered (Figure 1). In total, 37 of the 394 (9.3\%) randomly assigned participants did not receive the allocated intervention (Figure 1). The median (range) age at inclusion was
49.8 (40.7-55.8) years, 235 of 394 (59.6\%) participants were women, 260 of 393 (66.2\%) completed higher education, and 359 of 393 (91.3\%) were currently working. The mean (SD) duration of the current LBP episode was 7.5 (14.2) months, pain score was 41.7 (21.3), and QBPDI score was 29.3 (14.6). Overall, 157 of 197 (79.7\%) participants received the 6 sessions in the standard OMT group and 134 of 197 (68.0\%) in the sham OMT group (Table 1). A total of 20 trained osteopathic practitioners participated in the standard OMT and sham OMT sessions. Lumbar spine, root of mesentery, diaphragm, and atlantooccipital, sacroiliac, temporomandibular, and talocrural joints were treated in $94.0 \%, 88.6 \%, 86.3 \%, 81.0 \%, 60.9 \%$, $52.9 \%$, and $51.3 \%$ of the standard OMT sessions, respectively (eTable in Supplement 2).

\section{Primary Outcome}

Overall, 164 of 197 (83.2\%) patients in the standard OMT group and 159 of 197 (80.7\%) patients in the sham OMT group had primary outcome data available at 3 months. Nevertheless, all 394 participants had at least 1 measurement (baseline or postrandomization) that could be used for computing treatment effect differences. The mean (SD) QBPDI scores for the standard OMT group were 31.5 (14.1) at baseline and $25.3(15.3)$ at 3 months, and in the sham OMT group were $27.2(14.8)$ at baseline and 26.1 (15.1) at 3 months. At 3 months under the missing-data-at-random assumption, the mean reduction in LBP-specific activity limitations assessed via the QBPDI in the standard OMT and sham OMT groups was -4.7 (95\% CI, -6.6 to -2.8 ) and -1.3 (95\% CI, -3.3 to 0.6 ), respectively (mean difference in favor of standard OMT, -3.4 [95\% CI, -6.0 to -0.7$] ; P=.01$; Table 2). The cumulative proportion of responders analysis graph for patients with available QBPDI at 3 months is displayed in Figure 2 (post hoc sensitivity analysis). For an absolute change of at least 20 points in QBPDI score (ie, the minimal important change), the responder rate was $10.4 \%$ in the standard OMT group and $7.6 \%$ in the sham OMT group (absolute risk difference, 2.8 [95\% CI, -3.8 to 9.3]). 


\begin{tabular}{|c|c|c|c|c|c|}
\hline \multirow[b]{2}{*}{ Measurement } & \multicolumn{2}{|l|}{ No. $(95 \% \mathrm{Cl})$} & \multirow[b]{2}{*}{$\begin{array}{l}\text { Absolute difference, No. } \\
(95 \% \mathrm{Cl})^{\mathrm{b}}\end{array}$} & \multirow{2}{*}{$\begin{array}{l}\text { Relative risk or ratio } \\
\text { of means, No. ( } 95 \% \\
\text { CI) }\end{array}$} & \multirow[b]{2}{*}{$P$ value } \\
\hline & $\begin{array}{l}\text { Standard OMT group } \\
(n=197)\end{array}$ & $\begin{array}{l}\text { Sham OMT group } \\
(\mathrm{n}=197)\end{array}$ & & & \\
\hline \multicolumn{6}{|l|}{3 mo after randomization } \\
\hline Mean change in QBPDI score (range, $0-100)^{c}$ & $-4.7(-6.6$ to -2.8$)$ & $-1.3(-3.3$ to 0.6$)$ & $-3.4(-6.0$ to -0.7$)$ & NA & .01 \\
\hline Total reported & 164 & 159 & NA & NA & NA \\
\hline $\begin{array}{l}\text { At least } 20 \text { points improvement in QBPDI } \\
\text { score, No./total (\%) }\end{array}$ & $17 / 164(10.4)$ & $12 / 158(7.6)$ & $2.8(-3.8$ to 9.3$)$ & $1.4(0.7$ to 2.9$)$ & .41 \\
\hline $\begin{array}{l}\text { Mean change in LBP intensity on NRS (range, } \\
0-100)^{\mathrm{e}}\end{array}$ & $-8.1(-11.5$ to -4.7$)$ & $-7.1(-10.5$ to -3.7$)$ & $-1.0(-5.5$ to 3.5$)$ & NA & .66 \\
\hline Total reported & 165 & 159 & NA & NA & NA \\
\hline 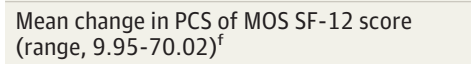 & $4.1(3.0$ to 5.3$)$ & $3.6(2.4$ to 4.8$)$ & $0.5(-1.0$ to 2.1$)$ & NA & .51 \\
\hline Total reported & 160 & 157 & NA & NA & NA \\
\hline $\begin{array}{l}\text { Mean change in MCS of MOS SF-12 score } \\
\text { (range, 5.89-71.97) }\end{array}$ & $1.7(0.3$ to 3.1$)$ & $1.2(-0.2$ to 2.6$)$ & $0.5(-1.4$ to 2.4$)$ & NA & .60 \\
\hline Total reported & 160 & 157 & NA & NA & NA \\
\hline Analgesic use, No./total (\%) & $49 / 162(30.3)$ & $39 / 154(25.3)$ & $4.9(-6.7$ to 16.5$)$ & $1.2(0.8$ to 1.8$)$ & .41 \\
\hline $\begin{array}{l}\text { Nonsteroidal anti-inflammatory drug use, } \\
\text { No./total (\%) }\end{array}$ & $29 / 169(18.0)$ & $31 / 158(19.6)$ & $-1.6(-11.1$ to 7.9$)$ & $0.9(0.6$ to 1.5$)$ & .74 \\
\hline \multicolumn{6}{|l|}{12 mo after randomization } \\
\hline Mean change in QBPDI score (range, $0-100)^{c}$ & $-5.6(-7.9$ to -3.3$)$ & $-1.3(-3.7$ to 1.2$)$ & $-4.3(-7.6$ to -1.0$)$ & NA & .01 \\
\hline Total reported & 136 & 121 & NA & NA & NA \\
\hline $\begin{array}{l}\text { Mean change in LBP intensity on NRS (range, } \\
0-100)^{\mathrm{e}}\end{array}$ & $-8.3(-12.1$ to -4.5$)$ & $-6.3(-10.3$ to -2.3$)$ & $-2.0(-7.2$ to 3.3$)$ & NA & .47 \\
\hline Total reported & 135 & 122 & NA & NA & NA \\
\hline 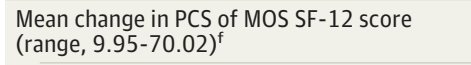 & $4.2(2.7$ to 5.6$)$ & 2.5 (1.0 to 3.9$)$ & $1.7(-0.3$ to 3.6$)$ & NA & .09 \\
\hline Total reported & 129 & 119 & NA & NA & NA \\
\hline 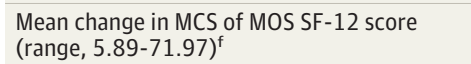 & $1.4(-0.2$ to 3.1$)$ & $2.5(0.8$ to 4.2$)$ & $-1.1(-3.3$ to 1.2$)$ & NA & .35 \\
\hline Total reported & 129 & 119 & NA & NA & NA \\
\hline Analgesic use, No./total (\%) & $43 / 129(33.3)$ & $35 / 117$ (29.9) & $3.4(-10.6$ to 17.5$)$ & $1.1(0.7$ to 1.8$)$ & .63 \\
\hline $\begin{array}{l}\text { Nonsteroidal anti-inflammatory drug use, } \\
\text { No./total (\%) }\end{array}$ & $21 / 135(15.6)$ & $25 / 120(20.8)$ & $-5.3(-15.8$ to 5.3$)$ & $0.7(0.4$ to 1.3$)$ & .32 \\
\hline Sick leave duration, mean (SD), d & $5.6(19.3)$ & $4.4(16.5)$ & $1.0(-3.2$ to 5.3$)$ & $1.4(0.4$ to 4.6$)$ & .58 \\
\hline Total reported & 136 & 130 & NA & NA & NA \\
\hline No. of sick leaves, mean (SD) & $0.3(0.7)$ & $0.2(0.5)$ & $0.0(-0.2$ to 0.2$)$ & $1.4(0.7$ to 2.5$)$ & .32 \\
\hline Total reported & 136 & 132 & NA & NA & \\
\hline No. of LBP episodes, mean (SD) & $11.8(17.5)$ & $14.8(19.9)$ & $-3.0(-7.7$ to 1.8$)$ & $0.8(0.6$ to 1.0$)$ & .08 \\
\hline Total reported & 120 & 108 & NA & NA & NA \\
\hline \multicolumn{2}{|c|}{$\begin{array}{l}\text { Abbreviations: LBP, low back pain; MCS, mental component summary; } \\
\text { MOS SF-12, Medical Outcomes Study Short Form 12; NA, not applicable; } \\
\text { NRS, numerical rating scale; PCS, physical component summary; } \\
\text { QBPDI, Quebec Back Pain Disability Index. }\end{array}$} & $\begin{array}{l}{ }^{\mathrm{c}} \text { Higher score } \\
{ }^{\mathrm{d}} \text { Post hoc ser }\end{array}$ & $\begin{array}{l}\text { idicate more limitations. } \\
\text { ivity analysis. }\end{array}$ & & \\
\hline
\end{tabular}

\section{Secondary Outcomes}

At 12 months, the mean reduction in LBP-specific activity limitations in the standard OMT and sham OMT groups was -5.6(95\% CI, -7.9 to $-3.3[\mathrm{n}=136])$ and $-1.3(95 \% \mathrm{CI},-3.7$ to 1.2 [n = 121]), respectively (mean difference in favor of standard OMT, -4.3 [95\% CI, -7.6 to -1.0 ]; $P=.01$ ). At 3 and 12 months, the mean difference in reduction in pain was $-1.0(95 \% \mathrm{CI},-5.5$ to $3.5 ; P=.66)$ and -2.0 ( $95 \% \mathrm{CI},-7.2$ to $3.3 ; P=.47)$, respectively. The standard OMT and sham OMT groups did not differ in mean improvement in HRQOL at 3 and 12 months, self-reported number and duration of sick leaves at 12 months, self-reported number of LBP epi- sodes at 12 months, or self-reported consumption of analgesics and nonsteroidal anti-inflammatory drugs since last contact at 3 and 12 months (Table 2).

\section{Safety}

At 12 months, 99 of 197 (50.3\%) participants in the standard OMT group and 93 of 197 (47.2\%) participants in the sham OMT group reported at least 1 adverse event. Twelve serious adverse events were reported in both groups: 4 in the standard OMT group (2 participants were hospitalized for another condition, and 2 received usual care for another condition) and 8 
Figure 2. Cumulative Percentage of Responders' Analysis With Change in Quebec Back Pain Disability Index (QBPDI) Score at 3 Months

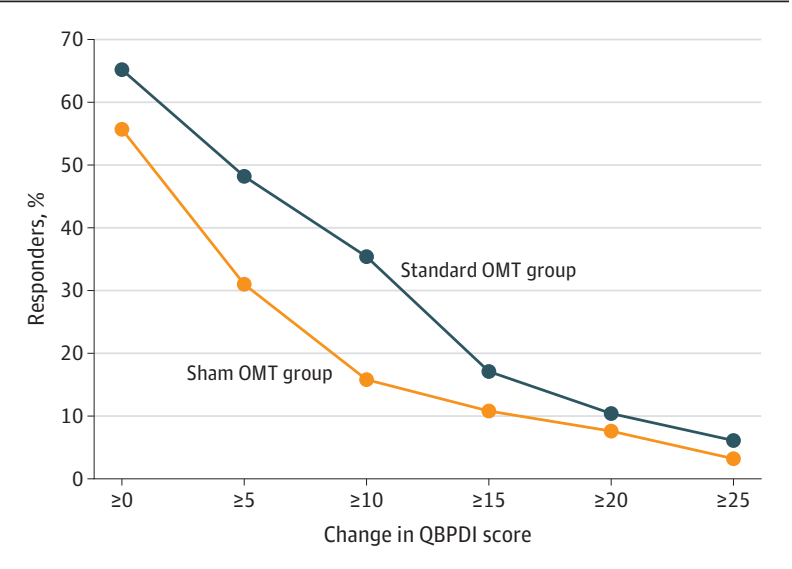

At 3 months, data were available for 164 participants in the standard osteopathic manipulative treatment (OMT) group and 159 in the sham OMT group.

in the sham OMT group (5 participants were hospitalized for another condition, 1 received usual care for LBP, and 2 received usual care for another condition). No serious adverse events were considered related to the interventions. Full details and absolute frequencies of minor and serious adverse events are reported in eAppendices 6 and 7 in Supplement 2, respectively.

\section{Credibility of the Interventions and Consistency}

of the Speech Content and Verbal Attitude

Scores for all 6 items of the Credibility/Expectancy Questionnaire were numerically higher in the standard OMT group than the sham OMT group (eAppendix 8 in Supplement 2). Of the 60 sessions randomly analyzed, the mean (SD) duration of sessions was 32.7 (6.0) minutes for the standard OMT group and 29.6 (3.5) minutes for the sham OMT group. Speech content and verbal attitude of osteopathic practitioners slightly differed for 4 of the 6 domains assessed (ie, instrumental exchanges, listening quality, reassurance, and relational proximity) (Figure 3). One osteopathic practitioner in each group mentioned their osteopathic training.

\section{Discussion}

As compared with sham OMT, standard OMT had a small effect on LBP-specific activity limitations at 3 and 12 months in patients with nonspecific subacute or chronic LBP. The clinical relevance of this effect is questionable. No evidence of difference was found between groups in pain, HRQOL, work absenteeism, or consumption of analgesics and nonsteroidal antiinflammatory drugs. No serious adverse event was considered related to OMT.

In the present study, LBP-specific activity limitations decreased in both groups, with a difference favoring standard OMT. The present population had low levels of LBP-specific activity limitations at baseline, which may in part explain the small magnitude of the effects we observed. In a metaanalysis of 2 RCTs $^{36,37}$ (1078 total participants) comparing the efficacy of manipulative treatment with other interventions for chronic LBP, manipulative treatment had a small positive effect on activity limitations at 3 months (standardized mean difference, -0.22 [95\% CI -0.36 to -0.07$]) .{ }^{10}$ As previously noted, comparisons with sham manipulative treatment were lacking, ${ }^{10}$ and comparators tested were "best care" 37 or "no intervention." ${ }^{36}$ Recently, in a cohort of 162 young adults with chronic LBP, Thomas and colleagues ${ }^{38}$ found no evidence of difference between manipulative treatment, mobilization, and placebo (sham cold laser) in reducing pain and disability in the short term.

In a systematic review, Rubinstein and colleagues ${ }^{9}$ compared the efficacy of manipulative treatment to sham manipulative treatment on LBP-specific activity limitations and did not find evidence of differences at $3^{36,39,40}$ and 12 months (3 RCTs with 573 total participants and 1 RCT with 63 total participants). Evidence was considered low to very low quality. When merging the present results with these findings, we found similar standardized mean difference values at 3 months ( -0.11 [95\% CI, -0.24 to 0.02]) and 12 months (-0.11 [95\% CI, -0.33 to 0.11 ) (4 RCTs with 896 total participants and 2 RCTs with 320 total participants).

Nonspecific OMT effects related to practitionerparticipant relationship, differences in health care providers' bedside manners, and treatment credibility could have contributed to the differences we observed. Indeed, Credibility/ Expectancy Questionnaire scores were numerically higher in the standard OMT group than the sham OMT group, as previously reported. ${ }^{41}$ They could indicate higher credibility of standard OMT vs sham OMT or higher expectations of participants. However, whether participants' expectations reflected failure of blinding, accurate assumptions about the efficacy of the interventions, or both is difficult to determine. ${ }^{42}$ Contextual factors could also have explained the differences we observed. Menke ${ }^{43}$ found that $81 \%$ and $66 \%$ of the variance in acute and chronic LBP, respectively, was because of manipulative treatment. The difference in the attention paid by the osteopathic practitioner when performing treatment could also play a role. ${ }^{44,45}$

We did not observe evidence of positive effects of standard OMT on pain at 3 and 12 months. One explanation could be the low level of initial pain. Evidence from 11 RCTs $^{10}$ (1894 total participants) indicated that manipulative treatment had a small effect on pain at 1 month. Consistent with the present findings, evidence from 10 RCTs (1587 total participants) and 7 RCTs (1728 total participants) showed that this effect was not maintained at 3 months (mean difference, -2.54 [95\% CI, -6.13 to 1.06]) or 12 months (mean difference, -0.89 [95\% CI, -2.92 to 1.14]), respectively. ${ }^{10}$ Finally, data for HRQOL and work status are sparse. In the present study, we did not observe evidence of positive effects of standard OMT on these outcomes.

\section{Limitations}

This study has limitations. We focused on standard OMT only. Some discrepancies with previous studies may be explained by the heterogeneity of manipulative treatments. ${ }^{10}$ The pre- 
Figure 3. Summary of Speech Content and Verbal Attitude of Osteopathic Practitioners According to Standard or Sham Osteopathic Manipulative Treatment (OMT)
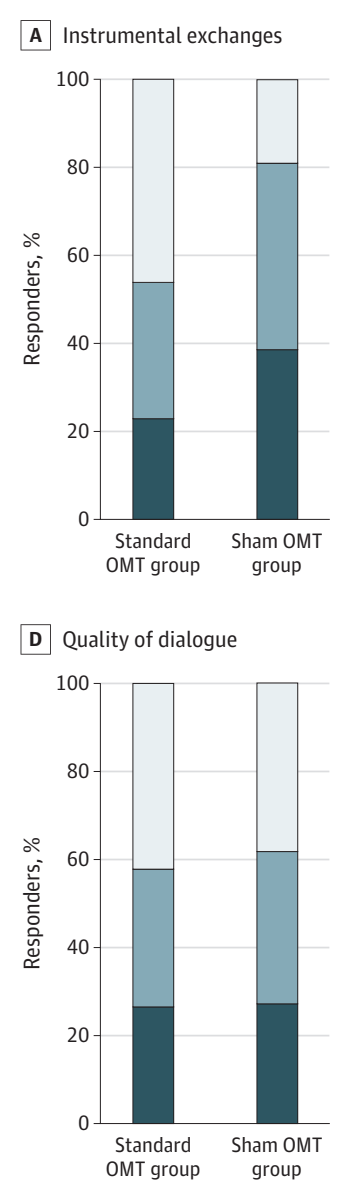

B Listening quality

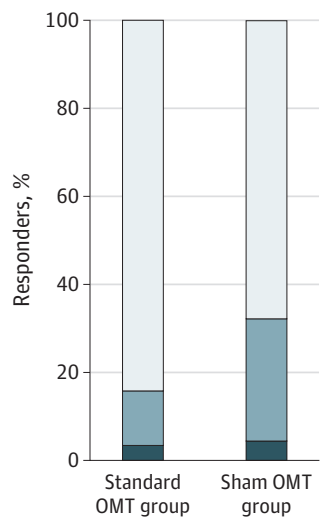

E Reassurance

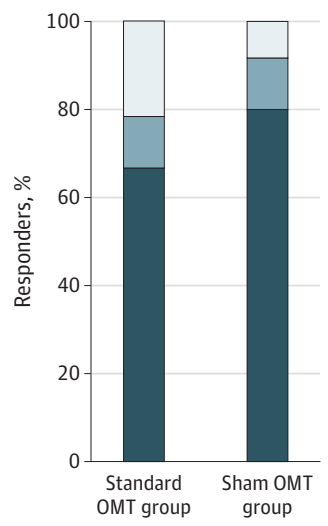

C Personal exchanges

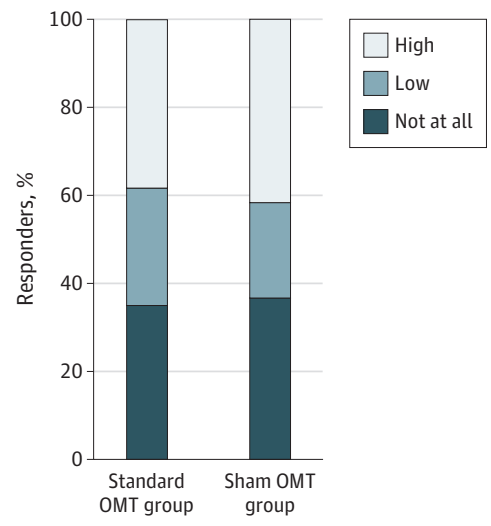

F Relational proximity

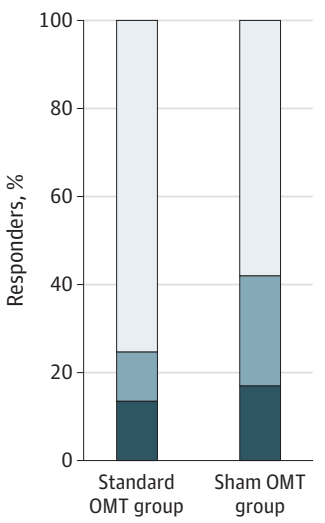

Scores range from 0 (not at all) to 2 (high). sent study population was recruited from a single center. Only nonphysician, nonphysiotherapist osteopathic practitioners were involved. Moreover, the validity of estimates of treatment effects from constrained longitudinal data analysis depends on missing-at-random data assumption; that is, the expected outcomes in patients with missing outcome data are assumed to be the same as observed outcomes among similar patients with nonmissing outcome data. This is a strong assumption that cannot be verified and is likely to be overoptimistic. The large loss to follow-up may have affected outcomes at 3 and 12 months. However, the missing-at-random assumption in this study could be reasonable. In fact, there was some evidence in this study that the probability of missingness for the primary outcome was related to higher baseline score values, which supports the missing-at-random assumption. Finally, measuring adverse events with an open question may have failed to fully capture adverse events because some patients may not have known what the term means.

\section{Conclusions}

In summary, in nonspecific subacute or chronic LBP, standard OMT vs sham OMT had a small effect on LBP-specific activity limitations. However, this effect was likely not clinically meaningful. For pain and absenteeism to work, no differences were observed. These results raise the issue of the usefulness of OMT in people with nonspecific subacute and chronic LBP.

\section{ARTICLE INFORMATION}

Accepted for Publication: January 1, 2021.

Published Online: March 15, 2021. doi:10.1001/jamainternmed.2021.0005

Author Affiliations: UFR de Médecine, Faculté de Santé, Université de Paris, Paris, France (Nguyen, Boutron, Daste, Lefèvre-Colau, Rannou); AP-HP.Centre-Université de Paris, Hôpital Cochin,
Service de Rééducation et de Réadaptation de l'Appareil Locomoteur et des Pathologies du Rachis, Paris, France (Nguyen, Sanchez, Daste, Boisson, Lefèvre-Colau, Rannou); INSERM UMRS-1124, Toxicité Environnementale, Cibles Thérapeutiques, Signalisation Cellulaire et Biomarqueurs (T3S), Campus Saint-Germain-des-Prés, Paris, France (Nguyen, Rannou); AP-HP.Centre-Université de Paris, Hôpital Hôtel-Dieu, Centre
d'Épidémiologie Clinique, Paris, France (Boutron, Baron); INSERM UMRS-1153, Centre de Recherche Épidémiologie et Statistique, METHODS Team, Paris, France (Boutron, Baron); A.T. Still Research Institute, A.T. Still University, Kirksville, Missouri (Zegarra-Parodi); COME Collaboration, Pescara, Italy (Zegarra-Parodi, Fabre); School of Health Sciences, HES-SO University of Applied Sciences and Arts Western Switzerland, Fribourg, 
Switzerland (Zegarra-Parodi, G. Krief); Cabinet d'Études Sociologiques Interlis, Paris, France (Alami); INSERM UMR-S 1153, Centre de Recherche Épidémiologie et Statistique, ECaMO Team, Paris, France (Daste, Lefèvre-Colau); Department Health Work Environment, Center for Primary Care and Public Health (Unisanté), University of Lausanne, Epalinges-Lausanne, Switzerland (P. Krief); Institut Fédératif de Recherche sur le Handicap, Paris, France (Lefèvre-Colau).

Author Contributions: Dr Nguyen and Profs Boutron and Rannou had full access to all of the data in the study and take responsibility for the integrity of the data and the accuracy of the data analysis.

Concept and design: Boutron, Zegarra-Parodi, P. Krief, Rannou.

Acquisition, analysis, or interpretation of dato: All authors.

Drafting of the manuscript: Nguyen, Boutron, Zegarra-Parodi, Baron, Fabre, G. Krief, Rannou. Critical revision of the manuscript for important intellectual content: All authors.

Statistical analysis: Baron.

Obtained funding: Boutron, Zegarra-Parodi, P. Krief, Rannou.

Administrative, technical, or material support: Sanchez.

Supervision: Nguyen, Boutron, Zegarra-Parodi Fabre, G. Krief, Rannou.

Other-assessment of the osteopaths' speech content and verbal attitude during the sessions with patients: Alami.

Conflict of Interest Disclosures: Prof Boutron reported receiving grants from the French Ministry of Health during the conduct of the study. $\mathrm{Mr}$ Zegarra-Parodi reported practicing as a registered osteopath in France and providing undergraduate and postgraduate courses in osteopathy. Dr Alami worked for Interlis and was hired by Assistance Publique-Hôpitaux de Paris to provide an assessment of the speech of the osteopathic practitioners. Mr Fabre reported practicing as a registered osteopath in France. Mr G. Krief reported practicing as a registered osteopath in Switzerland and providing postgraduate courses in osteopathy at the HES-SO-Fribourg. Prof Rannou reported receiving academic grants from Assistance Publique-Hôpitaux de Paris during the conduct of the study as well as grants from $\mathrm{H} 2 \mathrm{O} 2 \mathrm{O}$ and Programme Hospitalier de Recherche Clinique outside the submitted work. No other disclosures were reported.

Funding/Support: The study was funded by the French Ministry of Health (PHRC 2011, project P110142) and sponsored by the Département de la Recherche Clinique et du Développement de l'Assistance Publique-Hôpitaux de Paris.

Role of the Funder/Sponsor: The funders had no role in the design and conduct of the study; collection, management, analysis, and interpretation of the data; preparation, review, or approval of the manuscript; and decision to submit the manuscript for publication.

Data Sharing Statement: See Supplement 4.

Additional Contributions: We acknowledge Hendy Abdoul, MD, PhD, Alexandra Bruneau, PhD, Claire du Ranquet, MD, Farah Ketroussi, MSc, and Sabrina Leveau, MSc, of Unité de Recherche Clinique Centre d'Investigation Clinique Paris Descartes Necker/ Cochin for implementation, monitoring, and data management; Laura Smales for professional copy editing; Jacqueline Lamb for translating the original protocol from French to English: Marie Fechtenbaum, MD, MSc, Clémence Palazzo, MD, $\mathrm{PhD}$, and Guillaume Paris, MD, MSc, and residents from the Service de Rééducation et de Réadaptation de l'Appareil Locomoteur et des Pathologies du Rachis of Cochin Hospital in Paris, France for participation in participant enrollment; and all osteopathic practitioners for delivering interventions. Osteopathic practitioners received financial compensation, but others were not compensated for their contributions.

Additional Information: Serge Poiraudeau, MD $\mathrm{PhD}$, died on March 25, 2017. This article is dedicated to his memory. Prof Poiraudeau supported the idea, wrote the original protocol, obtained funding, and was the principal investigator of the trial. He closely supervised the project until his duties were taken over by Prof Rannou. In accordance with the journal policies, because he could not meet 2 of the 4 Internationa Committee of Medical Journal Editors authorship criteria (ie, final approval of the version to be published and agreement to be accountable for all aspects of the work in ensuring that questions related to the accuracy or integrity of any part of the work are appropriately investigated and resolved), Prof Poiraudeau could not be listed as an author and was removed at submission. We would like to acknowledge his huge contribution to this work and to honor his memory as an incredible researcher and colleague, a thoughtful mentor, and a close friend.

\section{REFERENCES}

1. GBD 2017 Disease and Injury Incidence and Prevalence Collaborators. Global, regional, and national incidence, prevalence, and years lived with disability for 354 diseases and injuries for 195 countries and territories, 1990-2017: a systematic analysis for the Global Burden of Disease Study 2017. Lancet. 2018;392(10159):1789-1858. doi:10. 1016/S0140-6736(18)32279-7

2. Qaseem A, Wilt TJ, McLean RM, Forciea MA Clinical Guidelines Committee of the American College of Physicians. Noninvasive treatments for acute, subacute, and chronic low back pain: a clinical practice guideline from the American College of Physicians. Ann Intern Med. 2017;166(7): 514-530. doi:10.7326/M16-2367

3. Nguyen C, Lefèvre-Colau MM, Kennedy DJ, Schneider BJ, Rannou F. Low back pain. Lancet. 2018;392(10164):2547. doi:10.1016/ S0140-6736(18)32187-

4. Carey TS, Evans AT, Hadler NM, et al. Acute severe low back pain. A population-based study of prevalence and care-seeking. Spine (Phila Pa 1976). 1996;21(3):339-344. doi:10.1097/00007632 199602010-00018

5. Waddell G. Simple low back pain: rest or active exercise? Ann Rheum Dis. 1993:52(5):317-319. doi 10.1136/ard.52.5.317

6. Bernstein IA, Malik Q, Carville S, Ward S. Low back pain and sciatica: summary of NICE guidance. BMJ. 2017;356:i6748. doi:10.1136/bmj.i6748

7. Chou R, Deyo R, Friedly J, et al. Systemic pharmacologic therapies for low back pain: a systematic review for an American College of Physicians clinical practice guideline. Ann Intern Med. 2017:166(7):480-492. doi:10.7326/M16-2458
8. The demography of osteopaths in France. Registre des Ostéopathes de France. Accessed February 7, 2021. https://www.osteopathie.org/ demographie.html

9. Rubinstein SM, de Zoete A, van Middelkoop M Assendelft WJJ, de Boer MR, van Tulder MW. Benefits and harms of spinal manipulative therapy for the treatment of chronic low back pain: systematic review and meta-analysis of randomised controlled trials. BMJ. 2019;364:1689. doi:10.1136/ bmj.1689

10. Rubinstein SM, van Middelkoop M, Assendelft WJ, de Boer MR, van Tulder MW. Spinal manipulative therapy for chronic low-back pain Cochrane Database Syst Rev. 2011;(2):CD008112.

11. Franke H, Franke JD, Fryer G. Osteopathic manipulative treatment for nonspecific low back pain: a systematic review and meta-analysis. $B M C$ Musculoskelet Disord. 2014;15:286. doi:10.1186/ 1471-2474-15-286

12. Geri T, Viceconti A, Minacci M, Testa M Rossettini G. Manual therapy: exploiting the role of human touch. Musculoskelet Sci Pract. 2019;44: 102044. doi:10.1016/j.msksp.2019.07.008

13. Paige NM, Miake-Lye IM, Booth MS, et al. Association of spinal manipulative therapy with clinical benefit and harm for acute low back pain: systematic review and meta-analysis. JAMA. 2017; 317(14):1451-1460. doi:10.1001/jama.2017.3086

14. Andersson GB, Lucente T, Davis AM, Kappler $\mathrm{RE}$, Lipton JA, Leurgans S. A comparison of osteopathic spinal manipulation with standard care for patients with low back pain. N Engl J Med. 1999;341(19):1426-1431. doi:10.1056/ NEJM199911043411903

15. Castro-Sánchez AM, Lara-Palomo IC Matarán-Peñarrocha GA, et al. Short-term effectiveness of spinal manipulative therapy versus functional technique in patients with chronic nonspecific low back pain: a pragmatic randomized controlled trial. Spine J. 2016;16(3):302-312. doi: 10.1016/j.spinee.2015.08.057

16. Task Force on the Low Back Pain Clinical Practice. American Osteopathic Association guidelines for osteopathic manipulative treatment (OMT) for patients with low back pain. J Am Osteopath Assoc. 2016;116(8):536-549. doi:10. 7556/jaoa.2016.107

17. Bolvig J, Juhl CB, Boutron I, et al; Editorial Board of the Cochrane Musculoskeletal Group. Some Cochrane risk-of-bias items are not important in osteoarthritis trials: a meta-epidemiological study based on Cochrane reviews. J Clin Epidemiol. 2018; 95:128-136. doi:10.1016/j.jclinepi.2017.11.026

8. Boutron I, Guittet L, Estellat C, Moher D, Hróbjartsson A, Ravaud P. Reporting methods of blinding in randomized trials assessing nonpharmacological treatments. PLoS Med. 2007; 4(2):e61. doi:10.1371/journal.pmed.004006

19. Puhl AA, Reinhart CJ, Doan JB, Vernon H. The quality of placebos used in randomized, controlled trials of lumbar and pelvic joint thrust manipulation-a systematic review. Spine J. 2017;17 (3):445-456. doi:10.1016/j.spinee.2016.11.003

20. Noll DR, Degenhardt BF, Fossum C, Hensel K. Clinical and research protocol for osteopathic manipulative treatment of elderly patients with pneumonia. J Am Osteopath Assoc. 2008;108(9) 508-516. 
21. Borkovec TD, Nau SD. Credibility of analogue therapy rationales. J Behav Ther Exp Psychiatry. 1972; 3:257-260. doi:10.1016/0005-7916(72)90045-6

22. Devilly GJ, Borkovec TD. Psychometric properties of the credibility/expectancy questionnaire. J Behav Ther Exp Psychiatry. 2000; 31(2):73-86. doi:10.1016/SOO05-7916(00)00012-4

23. World Health Organization. Benchmarks for Training in Traditional/Complementary and Alternative Medicine: Benchmarks for Training in Osteopathy. WHO Press; 2010.

24. Boutron I, Altman DG, Moher D, Schulz KF, Ravaud P; CONSORT NPT Group. CONSORT statement for randomized trials of nonpharmacologic treatments: a 2017 update and a CONSORT extension for nonpharmacologic trial abstracts. Ann Intern Med. 2017;167(1):40-47. doi: 10.7326/M17-0046

25. Hoffmann TC, Glasziou PP, Boutron I, et al. Better reporting of interventions: template for intervention description and replication (TIDieR) checklist and guide. BMJ. 2014;348:g1687. doi:10 1136/bmj.g1687

26. Kopec JA, Esdaile JM, Abrahamowicz M, et al. The Quebec Back Pain Disability Scale. Measurement properties. Spine (Phila Pa 1976). 1995;20(3):341-352. doi:10.1097/00007632 199502000-00016

27. Kopec JA, Esdaile JM, Abrahamowicz M, et al. The Quebec Back Pain Disability Scale: conceptualization and development. J Clin Epidemiol. 1996;49(2):151-161. doi:10.1016/ 0895-4356(96)00526-4

28. Yvanes-Thomas $M$, Calmels $P$, Béthoux $F$, et al. Validity of the French-language version of the Quebec back pain disability scale in low back pain patients in France. Joint Bone Spine. 2002;69(4): 397-405. doi:10.1016/S1297-319X(02)00415-3

29. Fitzmaurice GM, Laird NM, Ware JH. Applied Longitudinal Analysis. 2nd ed. John Wiley \& Sons; 2011.
30. Liang KY, Zeger SL. Longitudinal data analysis of continuous and discrete responses for pre-post designs. Sankhyā: Indian J Stat B. 2000;62(1).

31. Liu GF, Lu K, Mogg R, Mallick M, Mehrotra DV. Should baseline be a covariate or dependent variable in analyses of change from baseline in clinical trials? Stat Med. 2009;28(20):2509-2530. doi:10.1002/sim.3639

32. Ostelo RW, Deyo RA, Stratford P, et al. Interpreting change scores for pain and functional status in low back pain: towards international consensus regarding minimal important change. Spine (Phila Pa 1976). 2008;33(1):90-94. doi:10. 1097/BRS.0b013e31815e3a10

33. Farrar JT, Dworkin RH, Max MB. Use of the cumulative proportion of responders analysis graph to present pain data over a range of cut-off points: making clinical trial data more understandable. J Pain Symptom Manage. 2006;31(4):369-377. doi: 10.1016/j.jpainsymman.2005.08.018

34. Altman DG. Confidence intervals for the number needed to treat. BMJ. 1998;317(7168): 1309-1312. doi:10.1136/bmj.317.7168.1309

35. Bender R. Calculating confidence intervals for the number needed to treat. Control Clin Trials. 2001;22(2):102-110. doi:10.1016/ S0197-2456(00)00134-3

36. Licciardone JC, Stoll ST, Fulda KG, et al. Osteopathic manipulative treatment for chronic low back pain: a randomized controlled trial. Spine (Phila Pa 1976). 2003;28(13):1355-1362. doi:10. 1097/01.BRS.0000067110.61471.7D

37. Team UBT; UK BEAM Trial Team. United Kingdom back pain exercise and manipulation (UK BEAM) randomised trial: effectiveness of physical treatments for back pain in primary care. $B M J$. 2004;329(7479):1377. doi:10.1136/ bmj.38282.669225.AE

38. Thomas JS, Clark BC, Russ DW, France CR, Ploutz-Snyder R, Corcos DM; RELIEF Study
Investigators. Effect of spinal manipulative and mobilization therapies in young adults with mild to moderate chronic low back pain: a randomized clinical trial. JAMA Netw Open. 2020;3(8):e2012589. doi:10.1001/jamanetworkopen.2020.12589

39. Licciardone JC, Minotti DE, Gatchel RJ, Kearns CM, Singh KP. Osteopathic manual treatment and ultrasound therapy for chronic low back pain: a randomized controlled trial. Ann Fam Med. 2013;11 (2):122-129. doi:10.1370/afm.1468

40. Senna MK, Machaly SA. Does maintained spinal manipulation therapy for chronic nonspecific low back pain result in better long-term outcome? Spine (Phila Pa 1976). 2011;36(18):1427-1437. doi:10 1097/BRS.Ob013e3181f5dfeO

41. Licciardone JC, Russo DP. Blinding protocols, treatment credibility, and expectancy: methodologic issues in clinical trials of osteopathic manipulative treatment. J Am Osteopath Assoc. 2006;106(8):457-463.

42. Schulz KF, Altman DG, Moher D; CONSORT Group. CONSORT 2010 statement: updated guidelines for reporting parallel group randomised trials. BMJ. 2010;340:c332. doi:10.1136/bmj.c332

43. Menke JM. Do manual therapies help low back pain? a comparative effectiveness meta-analysis. Spine (Phila Pa 1976). 2014;39(7):E463-E472. doi: 10.1097/BRS.0000000000000230

44. Cerritelli F, Chiacchiaretta P, Gambi F, Ferretti A. Effect of continuous touch on brain functional connectivity is modified by the operator's tactile attention. Front Hum Neurosci. 2017;11:368. doi:10. 3389/fnhum.2017.00368

45. Cerritelli F, Chiacchiaretta P, Gambi F, et al. Effect of manual approaches with osteopathic modality on brain correlates of interoception: an fMRI study. Sci Rep. 2020;10(1):3214. doi:10.1038/ s41598-020-60253-6 